\title{
LA PRONUNCIACIÓN DEL ESPAÑOL EN LA RADIO DE VEINTE PAÍSES HISPÁNICOS: COMENTARIOS DEPORTIVOS ESPONTÁNEOS
}

\section{Antecedentes}

Hasta ahora se han descrito las variantes fónicas de cada país a través de la percepción de diferentes investigadores ${ }^{1}$, datos que posteriormente han sido recogidos por los lingüistas ${ }^{2}$. Las descripciones a las que me refiero se enfocan sobre todo en el habla popular, aunque más recientemente se ha incluido también la norma culta ${ }^{3}$, de nuevo a partir de los trabajos de diferentes investigadores (véase n. 5). En lo relativo a la radio, se ha mencionado su importancia en la promoción de la unidad lingüística, y por eso se han hecho propuestas relacionadas con la necesidad de que los locutores tengan una buena formación lingüística ${ }^{4}$. A pesar de esos señalamientos sobre la trascendencia y la influencia de la radio, no parece haber estudios sobre la pronunciación espontánea de los locutores por ese medio — menos aún cuando se trata de cubrir todos los países hispánicos. Tampoco ha habido un

${ }^{1}$ Agradezco ampliamente a Rodrigo Muñoz, de El Colegio de México, el apoyo que me dio en la recopilación y el tratamiento de los datos. Mi agradecimiento se extiende a Marianne Akerberg, quien —además de ayudarme a ampliar mi bibliografía- me apoyó en la interpretación de algunos fenómenos.

${ }^{2}$ Cf. M.C. RESNICK, Phonological variants and dialect identification in Latin American Spanish, Mouton, The Hague-Paris, 1975, y J.G. Moreno De Alba, El español en América, F.C.E., México, 1988, donde se incluye una extensa bibliografía al respecto.

3 Véase J.M. Lope Blanch, "La norma lingüística hispánica", ALM, 40 (2002), pp. 23-41.

${ }^{4}$ Véase J.M. Lope Blanch, La lengua española y sus problemas, UNAM, México, 1997, p. 43: en la "difusión de las normas lingüísticas ejemplares podrían desempeñar un buen papel la radio y la televisión, conforme han señalado Menéndez Pidal y Rafael Lapesa". Más adelante, Lope Blanch añade: "sería necesario que tanto periodistas como locutores de radio y televisión tuvieran una amplia y sólida formación gramatical y filológica, de manera que pudieran constituirse en buenos modelos idiomáticos" (p. 120). 
estudio hecho por un solo investigador ${ }^{5}$ sobre la pronunciación que se escucha en los países hispánicos. Esta investigación tiene como propósito llenar esos huecos ${ }^{6}$.

La importancia de la radio es evidente. Después de la imprenta, que promovió la lengua escrita ${ }^{7}$ y la democratización de la cultura en Europa, la radio fue el primer medio oral de difusión masiva, lo que le permitió superar la barrera del analfabetismo ${ }^{8}$. Al igual que la imprenta en relación con el léxico y la sintaxis, la radio ha difundido una pronunciación nacional o internacional estándar ${ }^{9}$, con base, en el caso del español, en las tres normas generales que he descrito previamente ${ }^{10}$ y que comento de nuevo más adelante. La pronunciación profesional (PP) de los locutores o comentaristas de la radio, además, resulta más apegada a la letra que la de los hablantes cultos, como he señalado en otro estudio ${ }^{11}$.

5 Una de las observaciones que hizo Manuel Alvar cuando presentó el Atlas lingüístico de México en El Colegio de México fue precisamente esa: que en el levantamiento de los mapas, sobre todo fonéticos, habían participado muchos investigadores: "pluralidad de exploradores y multiplicidad de informantes acrecientan los riesgos de la imprecisión" ("Ante el Atlas lingüístico de México”, NRFH, 39, 1991, sobre todo pp. 676 ss.).

6 N.S. TRubetzkoy advierte sobre la inadecuada percepción y los posibles errores de interpretación por causa del filtro o la criba fonológica de la lengua materna del investigador (Principios de fonología, Cincel, Madrid, 1973, pp. 46 y 56). Por mi parte, considero que lo más adecuado es analizar los dialectos de la lengua materna, pues se evita el filtro fonológico y no se cae en las limitaciones de quien investiga una lengua extranjera. En el caso del español en 20 países, el lingüista se enfrenta a variantes con alófonos suficientemente diferenciados, lo que facilita su percepción a partir de su dialecto nativo. En mi caso, ese dialecto es el español de México en su variante estándar, el cual tiene suficientes investigaciones para evitar mi propio sesgo o para usarlo como referente. Véase, entre otros, G. Perissinotto, Fonología del español hablado en la ciudad de México: ensayo de un método sociolingüístico, trad. R. Ávila, El Colegio de México, México, 1975.

7 Recordemos que el primer libro que publicó Gutenberg fue la Biblia, lo que promovió la reforma protestante encabezada por Lutero; véase, además, mi artículo “Qué español usar? La pronunciación en los comentarios de la radio”, Actas del VII Congreso Internacional de la Asociación Asiática de Hispanistas, Beijing, 2011, pp. 258-268.

8 Para mayores detalles sobre la importancia de la radio, véase Eulalio FERrER, Información y comunicación, F.C.E., México, 1997. Sobre el analfabetismo véase Hans R. DuA, Language planning in India, Harnan, New Delhi, 1985; sobre el papel de los medios en la planeación lingüística, p. 226, y en relación con la relevancia de la radio en el desarrollo y la estandarización de las lenguas, p. 238.

9 Para mayores detalles véanse David Crystal, The Cambridge encyclopedia of the English language, Cambridge University Press, Cambridge-New York, 1995 y RALPH Penny, Variation and change in Spanish, Cambridge University Press, Cambridge, 2000.

${ }^{10}$ Véase mi artículo “¿Qué español usar?” En ese estudio incluyo únicamente una estación de radio por cada una de las normas principales: seis en total. La presente investigación, en cambio, abarca las 20 estaciones de radio de mi muestra - como digo infra, una por cada capital hispánica.

11 En mi artículo "La pronunciación del español: medios de difusión masiva y norma culta” (NRFH, 51, 2003, pp. 57-79) hago referencia a esto. Considero que los 
Lo antes dicho sustenta la hipótesis de esta investigación: la radio promueve la internacionalización de la pronunciación del español. Esta propuesta se basa en los intereses propios del medio, sobre todo económicos y políticos ${ }^{12}$. Los primeros se relacionan con el nivel de audiencia, el llamado rating: si no se comprende lo que se transmite, no habrá audiencias, y mientras mejor se comprenda — sin considerar otros aspectos de contenido que no toco ahora- mayor será la cantidad de radioescuchas y el valor de los mensajes que se envíen. Por eso la radio utiliza en sus transmisiones una pronunciación que en inglés se conoce como received pronunciation $(\mathrm{RP})^{13}$, y que en español correspondería a lo que llamaré pronunciación comprendida por las audiencias (PCA).

Los aspectos políticos de las transmisiones de los medios se relacionan con los gobiernos de los países. La radio - como antes la imprenta- ha sido fundamental para la consolidación de los estados nacionales mediante la promoción de una sola lengua en todo el territorio ${ }^{14}$. Los gobiernos han buscado así la integración nacional de los diferentes grupos lingüísticos que conviven dentro de los límites del país. Los dialectos, aunque son formaciones anteriores a las de los estados nacionales, poco a poco han ido perdiendo sus límites a favor de los correspondientes a los países, áreas en las que los medios limitan en buena medida su influencia, sobre todo en relación con la radio. Tan evidente es el caso de los estados nacionales como fronteras lingüísticas, que - en lo relacionado con Hispanoamérica, por lo menos - para ir de uno a otro se requiere pasaporte, a diferencia de lo que sucedía en el siglo xix, cuyas fronteras dialectales eran otras ${ }^{15}$.

Mediante la imprenta, desde su nacimiento en el siglo Xv, se alcanzaba un propósito semejante, pero la letra impresa tenía, como he dicho supra (véase también n. 8), el problema del analfabetismo, que fue superado unos cinco siglos después, con la popularización de la radio antes de la Segunda Guerra Mundial. De lo anterior se desprende la necesidad de analizar grabaciones de conversaciones espontáneas a través de ese medio de difusión masiva, ya que cuando se leen,

locutores tienen una mejor articulación y, sin duda, una mejor voz que los hablantes cultos.

${ }^{12}$ Como indica Hans R. Dua (op. cit., p. 90), "the use of a standard language or a common national language may be necessary for the uniformity of message, mass production, and even its development and standardization".

13 Peter Trudgill ofrece información detallada de la RP en Sociolinguistic variation and change, Georgetown University Press, Washington, D.C., 2002.

${ }^{14}$ Véase Víctor Hugo Ramírez Ramírez, Lengua y diplomacia. La promoción lingüistica como elemento de política exterior, tesis, El Colegio de México, México, 2006.

${ }_{15}$ Véase, por ejemplo, Sever Pop, La dialectologie, Chez l'Auteur, Louvain, 1950, t. 1. Para Pop, los ríos unían y las montañas separaban las áreas dialectales. 
por ejemplo, las noticias, siempre existe la posibilidad del fetichismo de la letra, del referente escrito ${ }^{16}$.

La situación actual, con el gran desarrollo de las comunicaciones, en especial de internet ${ }^{17}$, y con los viajes cada vez más frecuentes, hace muy difícil —e incluso inconveniente para los medios como la radio y la televisión - la diversificación de la lengua. Ese temor, quizá originado en el siglo xix en Europa, con la extensión de la era industrial y las comunicaciones, no tiene sustento en el presente. Considérese además la expansión del sistema escolar y el abatimiento de los porcentajes de analfabetismo. Por eso resultan infundadas las inquietudes de algunos lingüistas que investigan la lengua española. No obstante sus preocupaciones, no estimulan suficientemente los estudios sobre los medios de comunicación masiva, más allá de algunas declaraciones y buenos deseos ${ }^{18}$. Es indudable el peso de la imprenta de tipos móviles en la estandarización de las lenguas europeas, así como el de la televisión y la radio, y más recientemente, de la internet. Sin embargo, sólo parecen haber merecido menciones, no estudios ${ }^{19}$.

\section{LA MUESTRA Y SU TRATAMIENTO}

Esta investigación se basa en 20 grabaciones de 30 minutos cada una ${ }^{20}$, correspondientes a una estación de radio de cada una de las 20 capita-

16 Véase Ángel Rosenblat, Nuestra lengua en ambos mundos, Salvat-Alianza, Barcelona-Madrid, 1971. Ese fetichismo es el que, en mi opinión, hace que los locutores de República Dominicana, cuando leen, articulen más frecuentemente el fonema /s/ en posición implosiva sin aspiración, como ocurre en la norma culta de ese país. Véase O. Alba, "Hipercorrección en los programas de noticias en la televisión de la República Dominicana”, en Variación del español en los medios, ed. R. Ávila, El Colegio de México, México, 2011, pp. 165-192.

17 Véase mi libro De la imprenta a la internet: la lengua española y los medios de comunicación masiva, $2^{a}$ ed., El Colegio de México, México, 2009, sobre todo el cap. 9. Hay una descripción extensa del lenguaje de la red mundial en D. Crystal, Language and the Internet, $2^{\mathrm{a}}$ ed., Cambridge University Press, Cambridge, 2006. Como era de esperarse, la mayor parte del contenido se refiere a la lengua inglesa, pero hay algunos ejemplos de nivel internacional que atañen al español y, por supuesto, a muchas otras lenguas.

18 Entre otros, J.M. Lope Blanch. En su art. cit., p. 25, dice que es necesario "esforzarnos para mantener la unidad fundamental de la lengua", y que debemos "tratar de evitar... la «evolución diversificadora» que tanto preocupaba a Dámaso Alonso”. Y continúa: "para lograr esa homogeneidad lingüística entre las hablas de 20 países soberanos... la aceptación, por parte de todos, de una norma hispánica general sería condición muy favorable" (p. 26).

19 Menciones que, por cierto, muestran a veces preocupaciones. Recuérdense las sugerencias de Lope BlANCH (La lengua española..., pp. 43 y 120), citadas supra, nota 4.

20 Hubo un mínimo de dos y un máximo de tres comentaristas (véase Apéndice). Además, se hicieron entrevistas a personas que hablaban por teléfono a las distintas estaciones de radio. Las entrevistas, sin embargo, no fueron consideradas en esta investigación. Tampoco se tomaron en cuenta los anuncios publicitarios. 
les de los países hispánicos ${ }^{21}$. Los comentaristas — todos de sexo masculino- hablaron en forma espontánea ${ }^{22}$ de un tema que no se buscó a priori, sino que se encontró en todas las estaciones: deportes, sobre todo futbol —aunque también se habló de beisbol en algunas transmisiones de radio del área del Caribe. El estilo que emplearon los comentaristas puede calificarse como coloquial público $^{23}$. Las grabaciones fueron recogidas a través de internet, a fines de 2008 y principios de 2009. Posteriormente, hicimos nuevas grabaciones en el año 2015. En esta nueva audición no encontré grandes diferencias con respecto a la anterior, con excepción de las estaciones de radio de las capitales de Ecuador y Bolivia, cuyos locutores ya no hacían la distinción y/ $\lambda$ como en las grabaciones previas de 2008 y 2009.

Las 20 grabaciones fueron transcritas digitalmente de acuerdo con los usos ortográficos actuales, ya que se hicieron análisis léxicos y sintácticos. En cambio, los aspectos fonético-fonológicos se recogieron mediante la audición cuidadosa de cada una. La audición y las transcripciones —como mencioné supra-fueron hechas por un solo investigador: el autor de este artículo ${ }^{24}$. Esto permite superar lo que he comentado anteriormente en relación con los investigadores y sus sesgos (notas 5 y 6 ). Reitero ahora que las variantes fónicas del español que transmite la radio son lo suficientemente diferenciadas como para evitar que mi dialecto nativo me condicione y que, además, el español de México ha tenido suficientes descripciones fonéticas como para utilizarlo, en todo caso, como referente fonético-fonológico ${ }^{25}$.

El tema de comentarios deportivos — como dije supra- no fue seleccionado por nosotros, sino que apareció en todas las estaciones, lo que aseguró una comparación homogénea. Tampoco fue

${ }^{21}$ De aquí en adelante utilizaré "países (hispánicos)" y "estaciones (de radio)" respectivamente, como términos equivalentes.

${ }^{22}$ La excepción fue quizá La Habana, donde los comentaristas daban la impresión de estar leyendo, aunque pude recoger una conversación espontánea en 2015.

${ }^{23}$ Antonio María LóPez GonzÁlez señala en su tesis de doctorado, El español en la radiodifusión de la zona metropolitana de Almería (Universidad de Almería, 2001) que, conforme era más formal la transmisión — por ejemplo, lectura de noticias- más se acercaba el estilo de los locutores a la norma castellana. Véase la descripción de esa norma más adelante, y también la Figura 1.

${ }^{24}$ En los muy raros casos en que tuve dudas, pedí a dos de mis colegas que escucharan las grabaciones. Además, para la casi totalidad de las variantes conté con la confirmación que ofrecía la bibliografía al respecto, como puede verse más adelante, en notas. Mis datos - como los de los Atlas y de la mayoría de las investigaciones dialectales - se pueden considerar impresionistas en la medida en que no he utilizado más recurso que mi oído, como han hecho muchos otros investigadores. Reitero que casi todos ellos se apoyan en datos bibliográficos, pero no en audiciones directas, como lo hice yo en el caso de la radio.

${ }^{25}$ Me he basado únicamente en los aspectos segmentales, sin considerar la entonación. El nivel segmental ha sido suficiente para establecer una clasificación diferencial cualitativa de la pronunciación de la radio por países, con unas pocas excepciones, en las cuales tuve que recurrir a aspectos cuantitativos (véanse Figuras 2 y 3 ). 
seleccionado el sexo de los locutores (todos fueron hombres) ${ }^{26}$, lo que corresponde a una base de comparación sin la variante sexo. Los comentaristas, sin duda, sabían que las transmisiones se hacían también por internet, lo que les hacía tomar conciencia, de alguna manera, del español que estaban usando. Esto los llevaba a emplear, por ejemplo, un léxico internacional, con pocos términos marcados o regionales, como he mostrado en otras investigaciones ${ }^{27}$.

Mi análisis, a diferencia de otros, hace explícita la variante en la que se basa. Sucede frecuentemente que los lingüistas proponen diferenciaciones dialectales pero no especifican, porque tal vez no se encuentra en la bibliografía consultada, el estilo ni el nivel sociocultural en el que se basan para sus descripciones ${ }^{28}$. También llama la atención que no se hable de los medios y la necesidad de analizar el empleo del español con base en un corpus cuando se mencionan los problemas de unidad y diversidad del español en el mundo.

\section{LAS NORMAS INTERNACIONALES DE PRONUNCIACIÓN ${ }^{29}$}

Las normas que se escuchan por la radio son de tres tipos, que en anteriores trabajos he llamado alfa $(\alpha)$, beta $(\beta)$ y gama $(\gamma)$. El orden y la clasificación de esas normas responden a la frecuencia con que se utilizan en las transmisiones por televisión de alcance internacional ${ }^{30}$. Dado que he publicado varios artículos referidos a esas normas, decidí no modificarlas en esta ocasión, porque no era necesario. Su descripción, tanto para la televisión como para la PP de la radio, en una primera instancia, es la siguiente: en la norma alfa no se distinguen

26 Los comentaristas eran nativos de cada país o de cada ciudad desde donde se hacían las transmisiones, de acuerdo con nuestras propias investigaciones. Pueden verse sus nombres en el Apéndice, junto con otros datos de las estaciones radiodifusoras.

27 Véase, para ejemplos léxicos, mi libro citado De la imprenta a la internet, sobre todo pp. 131 ss. y pp. 164 ss.

28 Esto parece ser la constante, como puede advertirse desde Alonso ZamorA Vicente, Dialectología española, Gredos, Madrid, 1960. Más recientemente, puede verse, entre otros, José IgnaCio HuAlde, The sounds of Spanish, Cambridge University Press, Cambridge-New York, 2005, cap 2. En esas páginas HuAldE hace referencia a los sociolectos y a los estilos del español en el mundo, pero sólo los declara, no los describe. Algo semejante se encuentra en John M. LiPski, Latin American Spanish, Longman, London-New York, 1994. De paso, en relación con la terminología, para la invención de Latinoamérica en el siglo XIX, véase J.M. Lope BLANCH, "Latinoamérica, Iberoamérica, Hispanoamérica”, en Actas del X Congreso Internacional de la ALFAL, Veracruz, México, del 11 al 16 de abril de 1993, UNAM, México, 1996.

29 Para las trascripciones utilizo el Alfabeto Fonético Internacional o IPA, por su sigla en inglés (International Phonetic Alphabet). La versión 2005, en la que me baso, puede encontrarse mediante cualquier buscador de internet.

30 Para una descripción previa de estas tres variantes, véase mi artículo, ya citado, "La pronunciación del español: medios de difusión masiva y norma culta". 
los fonemas /s/ y / $\theta /$, y no se aspira el fonema /s/ implosivo; en la norma beta, además de la no distinción de los fonemas $/ \mathrm{s} / \mathrm{y} / \theta /$, se aspira el fonema /s / en posición implosiva; y en la norma gama, por último, se hace la distinción entre $/ \mathrm{s} / \mathrm{y} / \theta /$, y no se aspira el fonema /s/ en posición implosiva (ver Figura 1).

FIGURA 1.

\begin{tabular}{|l|c|c|}
\hline & $\theta \neq \mathbf{s}$ & $\mathbf{s}>\mathbf{h}$ \\
\hline 1 alfa & no & no \\
\hline 2 beta & no & sí \\
\hline 3 gama & sí & no \\
\hline
\end{tabular}

Diferencias iniciales alfa, beta y gama.

Estas diferencias permiten establecer tres isoglosas generales por países, las cuales matizaré a continuación. Añado que, a diferencia de lo que ocurre con otras descripciones ${ }^{31}$, la mía se basa en los rasgos pertinentes desde el punto de vista diferencial de las variantes ${ }^{32}$.

El referente de la lengua escrita ${ }^{33}$ es inevitable incluso cuando se trata de los medios orales. En ausencia de un texto impreso, la escritura sigue en la mente de los hablantes alfabetizados ${ }^{34}$. En relación con la ortografía, la norma gama utiliza, para la transcripción del fone$\mathrm{ma} / \mathrm{z} /$, las grafías $\langle\mathrm{c}>$ ante $<\mathrm{e}\rangle,<\mathrm{i}\rangle$, y en los demás casos $<\mathrm{z}>$. Esto no ocurre con las normas alfa y beta, que no diferencian el sonido de esas

31 Véanse, por ejemplo, los trabajos citados de Moreno de Alba y Hualde. La preocupación de esos autores no parece ser la de diferenciar las variantes, sino sólo de acumular sus características incluso con rasgos redundantes. Como resultado, hay dialectos no adecuadamente caracterizados desde el punto de vista fonéticofonológico. Lo mismo hace JoHn Lipski (op. cit.), entre otros.

32 Véase supra n. 24. Por su parte, J.P. RonA, "El problema de la división del español americano en zonas dialectales", en Presente y futuro del español, Cultura Hispánica, Madrid, 1964, t. 1, pp. 222 ss., hace una clasificación de las zonas dialectales del español americano con base en rasgos no redundantes. Sin embargo, no son suficientemente diferenciadores. Compárense las zonas 2 y 4, y 17 y 18 , las cuales resultan idénticas en la clasificación que propone el autor. Dada la jerarquización de los rasgos que utilizo, considero necesario el empleo del término taxonomía en lugar de hablar de una clasificación.

33 Véanse al respecto el art. cit. de O. Alba y el libro cit. de A. Rosenblat, así como mis comentarios en relación con los locutores de la República Dominicana (n. 16).

34 Por ejemplo, los mexicanos en su mayoría dicen [sanória], pero cuando se les pide que silabeen la palabra, la pronuncian [sa-na-ó-ria], de acuerdo con su forma escrita <zanahoria > Recuerdo, además, que un niño de una escuela primaria de La Habana, cuando leía lo hacía prácticamente como mexicano (norma alfa más -n), pero cuando hablaba, lo hacía como habanero, de acuerdo con la pronunciación que era natural para él. Véase también lo que he comentado en relación con el art. cit. de O. Alba y la op. cit. de Rosenblat en nota 16. 
letras del de la $<\mathrm{s}>$. Asimismo, el grafema $<$ ll $>$ permite diferenciar países (ver infra).

Las estaciones de radio capitalinas — ciudades como Madrid, México, Caracas, Bogotá, Lima o Buenos Aires- transmiten en las tres normas que he mencionado. La norma alfa se escucha en las estaciones de radio de las capitales de México, Bolivia, Ecuador, Colombia, Guatemala, El Salvador y Costa Rica. En la PP de esos países, además, es posible distinguir entre la variante alfa 1 , donde el fonema / $\mathrm{x} /$ se pronuncia palatal fricativo (México, Bolivia, Ecuador), y alfa 2, donde se abre y se relaja ese mismo fonema (Colombia, Guatemala, El Salvador, Costa Rica). En la norma alfa 1 es posible distinguir entre la PP de Bolivia y la de Ecuador ${ }^{35}$, pues en las grabaciones de 2009 se escuchaba una diferenciación de los fonemas $/ \mathrm{y} / \mathrm{y} / \lambda /{ }^{36}$, pero no en las de 2015. En estas últimas grabaciones es suficiente señalar que tanto en Ecuador como en Bolivia se pronuncia [3], lo que no ocurre en México. Para diferenciar los dos países andinos basta indicar que en el primero el fonema /s/ se pronuncia sonoro al final de palabra ante vocal, como en [dosempúnto] (“doce en punto”) y [dozempúnto] ("dos en punto") ${ }^{37}$.

Frente a los países anteriores, en las estaciones de radio de Colombia, Guatemala, El Salvador y Costa Rica el fonema /x/, como he indicado antes, se pronuncia faríngeo, con una mayor o menor apertura, variante que transcribiré con [ $\hbar]$. Por otra parte, en Colombia no recogí la pronunciación velar [y] de /n/ final de palabra, la que sí se presenta en los demás países. La diferencia entre El Salvador, Costa Rica y Guatemala consiste en que en las primeras dos estaciones de radio escuché una pronunciación relajada de $/ \mathrm{b} / / \mathrm{d} / \mathrm{y} / \mathrm{g} /{ }^{38}$ entre vocales, la cual, comparativamente, los distingue de Guatemala. Entre Costa Rica y El Salvador la diferencia está marcada por la pronunciación aproximante $[\mathrm{I}]$ del fonema / $\mathrm{f} /$ en el primer país.

Las diferencias entre las estaciones de radio que transmiten en norma beta, permiten distinguir entre los países beta del norte y del sur de América; he clasificado como beta 1, los del norte, frente a beta 2 y beta 3, los del sur. La pronunciación faríngea [ $\hbar]$ del fonema /x/ caracteriza a los países del primer grupo, donde se escucha usualmen-

35 Cf. H. Toscano Mateos, "El español hablado en el Ecuador", en Presente y futuro de la lengua española, t. 1, p. 115.

36 Así lo ratifican Moreno de Alba (op. cit., p. 149) y D.L. CANField, La pronunciación del español en América (Instituto Caro y Cuervo, Bogotá, 1962), mapa 5. Véase además P. Boyd-Bowman, "Sobre la pronunciación del español en el Ecuador", NRFH, 7 (1953), p. 224. Véase también JoHn M. LiPski, Latin American Spanish, Longman, London, 1994, p. 188 para Bolivia, y p. 248 para Ecuador. Reitero que he recogido la pronunciación de las estaciones de radio de las ciudades capitales, la que no necesariamente corresponde a lo que se escucha en todas las regiones de cada país.

37 Cf. Lipski, op. cit., y Moreno de Alba, op. cit., entre otros.

38 Véase Melvyn C. Resnick, op. cit., pp. 20 (número de índice C2) y 86-87. 
te en la PP de la radio, frente a los del segundo y el tercero, donde no se escucha.

Los países beta 1 son los que se encuentran en el área del Caribe: Venezuela, Panamá, Cuba, República Dominicana, Puerto Rico, Nicaragua y Honduras ${ }^{39}$. En la República Dominicana y en Puerto Rico, además, los fonemas /b/, /d/ y /g/ entre vocales se pronuncian relajados, lo que no ocurre con la misma frecuencia e intensidad, comparativamente, en los demás países. Entre Dominicana y Puerto Rico la diferencia se puede establecer por la baja y la alta frecuencia de la pronunciación lateralizada del fonema / $\mathrm{r} /$ implosivo.

\section{FIGURA 2.}

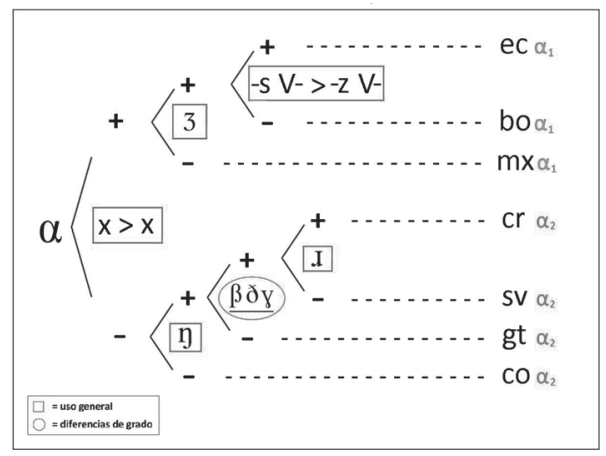

Taxonomía países alfa.

Los rasgos diferenciadores de Venezuela, Panamá, Cuba, Nicaragua y Honduras son los siguientes: el fonema / $\mathrm{j} /$ se pronuncia abierto [j] en Panamá, Nicaragua y Honduras, y con menor apertura en Venezuela y Cuba, países que se diferencian por la pronunciación relajada de /d/ entre vocales en el primero, lo que no es tan frecuente en el segundo.

En relación con Panamá, Nicaragua y Honduras, en el primer país es muy frecuente la relajación del fonema /d/ en posición intervocálica, lo que no ocurre tan consistentemente en los otros dos países. La distinción entre la PP de Honduras y de Nicaragua estriba en que en Honduras el fonema /s/ se escucha con un sonido sonoro asimilado a /b/, /d/, /g/, como en <es buena $>$ [e $\mathrm{e}^{\mathrm{b}}$ buéna], $<$ los gatos $>$ [logátoh] y $<$ desde aquí $>$ $\left[\text { de }^{\text {ddeakí }}\right]^{40}$, lo que no recogí en Nicaragua (ver Figura 3).

39 Sin embargo, otros países que tienen costa en esa región no son beta 1 , sino alfa, de acuerdo con la PP de las transmisiones de radio de sus capitales, como Colombia, México y Guatemala (ver su descripción supra y Figura 2).

40 Véanse Lipski, op. cit., Moreno de Alba, op. cit. y Hualde, op. cit. En relación con Honduras (entre otras "parts of Central America”, p. 28), este último detalla que /b/, /d/ y /g/ se pronuncian oclusivas después de cualquier consonante. En mi descripción, cuando la consonante posterior a /s / es /b/, /d/o /g/, la pronunciación del grupo resulta geminada, lo que recogí sólo en la PP de Honduras. Véase también Canfield, op. cit., mapa 1. 
Los países beta de Suramérica -Perú, Paraguay, Argentina, Uruguay y Chile- se distinguen de los del norte, como indiqué supra, porque la pronunciación del fonema / x/ es velar, no faríngea. En la PP de esos países escuché las siguientes diferencias: en Perú se pronuncia la variante velar $[\mathrm{\eta}]$ como alófono de / $\mathrm{n}$ / en posición final de palabra ante vocal. La palatal lateral [ $\lambda$ ] se escucha en Paraguay, pero no en Argentina, Uruguay o Chile. En Argentina y Uruguay se escucha, para el fonema $/ \mathrm{j} /$, la variante [3], lo que no ocurre en Chile ${ }^{41}$. Entre Argentina y Uruguay la diferencia que pude establecer se relaciona con la realización ensordecida [ $\left.\int\right]$ del mismo fonema, que resulta más frecuente en Argentina $^{42}$ que en Uruguay (Figura 3).

En cuanto al único país con PP gama, España (Figura 3), la diferencia consiste, frente a todos los demás países, en que los comentaristas deportivos madrileños distinguen los fonemas /s/ y / $\theta /$, como dije antes. La oposición fonológica, en este caso, es de sistema, si se considera que cuando en un conjunto de elementos no está presente uno de ellos, se trata de otro sistema ${ }^{43}$.

FIGURA 3.

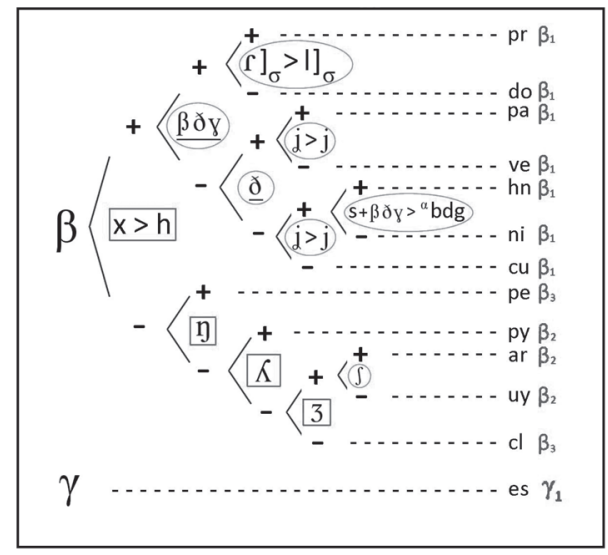

Taxonomía países beta y gama.

41 Véanse Lipski, op. cit. y Moreno de AlbA, op. cit., entre otros.

42 Esto se confirma con la sorpresa que le causó a Lope BLANCH (La lengua españo$l a \ldots$, p. 22 y n. 27) y a otras personas hispanohablantes escuchar a un argentino decir [ Jo féßo la fáße] <yo llevo la llave>, con ensordecimiento del fonema palatal. Lo mismo menciona Lope Blanch, con el mismo ejemplo, en p. 44. Véanse también pp. 45 y 46.

43 Francisco Moreno Fernández, en sus libros Qué español enseñar, Arco/Libros, Madrid, 2000 y Las variedades de la lengua española y su enseñanza, Arco/Libros, Madrid, 2010, coincide con mis apreciaciones. Sin embargo, Moreno Fernández no procede por países, sino por regiones. Además de tener una finalidad distinta, el autor parece basarse en sus abundantes datos bibliográficos, pero no necesariamente en una audición directa de la mayoría de las variedades, o no lo menciona. 


\section{Conclusiones}

Es indudable la importancia que han tenido los medios de comunicación o difusión masiva - de la imprenta a la internet- en la estandarización de las lenguas europeas. Los medios siempre han respondido a los intereses de los estados nacionales ${ }^{44}$, aunque en la actualidad expresan cada vez más los de las empresas transnacionales.

Gracias a la imprenta se consiguió estandarizar, mediante la publicación de la Biblia, las lenguas del norte de Europa. No obstante, el analfabetismo fue el gran obstáculo al que se enfrentó la lengua escrita. Esta limitante fue superada siglos después por la radio, el primer medio de difusión oral de las lenguas. Más tarde la televisión, de vocación internacional, promovió de nuevo la unidad lingüística. En la actualidad, la internet, de vocación mundial, permite no sólo la transmisión de la lengua escrita, sino también de la hablada —además, por supuesto, de imágenes y videos. De manera inevitable, la red mundial requiere la utilización de una norma lingüística internacional.

Por lo anterior, llama la atención el hecho de que, aparte de las declaraciones al respecto, no se hayan hecho investigaciones sobre el español de la radio —o de la televisión o la internet- en el ámbito internacional. Habría que pasar de las declaraciones a las investigaciones básicas y teóricas. La que presento - aunque tiene sus propias limitaciones- fue hecha directamente por un solo investigador, lo que permite superar los planteamientos generales que se sustentan, casi sin excepción, en referencias bibliográficas de diferentes estudiosos.

Mis resultados se basan en la audición de la PP de 20 estaciones de radio: una por cada capital hispánica. La PP de los locutores ofrece la posibilidad de establecer una taxonomía de países a partir de rasgos distintivos, no redundantes o cualitativos. Sin embargo, en algunos casos he recurrido a aspectos de tipo cuantitativo, de acuerdo con la mayor o menor frecuencia de uso de un determinado alófono (ver Figuras 2 y 3). Los resultados muestran que hay una gran unidad fonética ${ }^{45}$ del español a nivel internacional a partir de las tres normas que he descrito: la alfa, seseante y sin aspiración de /s/ implosiva; la beta, seseante y con aspiración de /s/ en la misma posición; y la gama, ceseante y sin aspiración de /s/ final de sílaba.

He comentado que la televisión de alcance internacional promueve la unidad lingüística del español. Lo mismo ocurre con la radio en el nivel nacional, por motivos económicos y políticos, lo que confir-

${ }^{44}$ Véase X.C. Lagares, “O espaço político da língua espanhola no mundo”, TLA, 52 (2013), pp. 385-408. En p. 391, LAGARES señala que la idea de lengua nacional, que surge en Europa a partir del siglo XIx, requiere que esa lengua sea "necessariamente homogénea e estável, como principal instrumento de unidade política".

${ }^{45}$ Para la pronunciación en los medios, véase mi libro citado De la imprenta a la internet, sobre todo el cap. 7.3. 
ma la hipótesis que propuse en esta investigación. En la actualidad los países, como límites de los estados nacionales, delimitan las normas idiomáticas a través de los medios y del sistema escolar, cada vez más extendidos. Este hecho implica que los dialectos poco a poco van dejando su lugar a las formaciones lingüísticas nacionales o supradialectales $^{46}$, aunque algunas isoglosas rebasen los límites de los países. Resulta claro que la regulación de la forma de hablar de los hispanohablantes tiene que ver — más que con instituciones- con la sanción o el consenso sociales. Y eso es lo que, en mi opinión, han tomado en cuenta los medios de difusión masiva: la pronunciación comprendida y aceptada por las audiencias (ver Figura 4). De manera inevitable, por su influencia y su prestigio, los medios orales, como antes la imprenta, están generando el consenso social en relación con las variantes fónicas de la lengua española. Y al hacerlo, promueven la unidad internacional de la lengua.

\section{FigURA 4.}

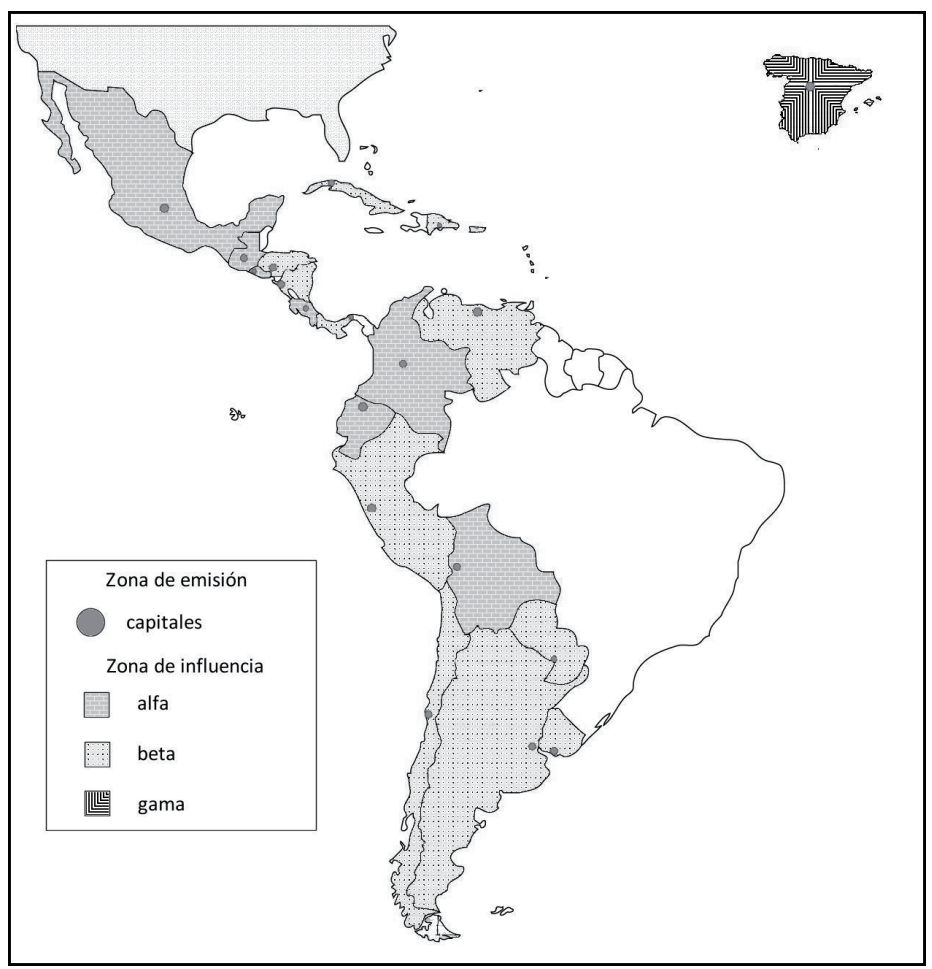

Zonas de emisión y de influencia (PCA).

46 Por ejemplo, en Italia, a fines del siglo xIx, sólo el 10\% de la población hablaba italiano. En la actualidad, gracias a la escuela y a los medios, en todo el país se requiere aprender el italiano como lengua nacional. Véase para esto Tulio Di MAuro, Storia linguistica della Italia unita, Laterza, Roma, 1983. 
Por eso considero que las preocupaciones de algunos lingüistas en relación con la diversificación del español no parecen justificarse en la actualidad. Se explicarían, quizás, a partir de la expansión industrial del siglo XIX, que impuso normas lingüísticas generales y logró que se perdieran algunas lenguas minoritarias. El auge actual de las comunicaciones - que da, por ejemplo, la posibilidad de que en los hogares se escuchen las normas internacionales-, la generalización de la alfabetización y del sistema escolar y los constantes viajes a otros países promueven la unidad lingüística de la lengua española.

Lo anterior lleva a discutir la supuesta hegemonía de una variante: la gama, del norte-centro de España ${ }^{47}$. Mis datos muestran que esa norma es la única que no rebasa el ámbito nacional. Si se considera el número de hablantes - el mercado potencial para esa pronunciación-, el de la norma gama resulta indudablemente minoritario en comparación con las audiencias de las normas alfa y beta. Por eso los medios orales, la televisión y la radio sobre todo, utilizan esas dos normas en sus producciones y doblajes ${ }^{48}$, principalmente la alfa.

En todo caso, los lingüistas deben tomar en cuenta, necesariamente, a todos los actores que intervienen en la actualidad en la difusión y estandarización de la lengua española. Deben advertir, más allá de las descripciones puramente lingüísticas y de las declaraciones, los intereses de los gobiernos nacionales, de las grandes empresas nacionales y transnacionales y, dentro de ellas, los de los medios de difusión masiva y su mercado lingüístico.

\author{
Raúl Ávila \\ El Colegio de México
}

\footnotetext{
47 Véase el art. cit. de Lagares, pp. 399 ss.

${ }^{48}$ Véase mi artículo "Los increíbles: doblajes y subtítulos", en Estudios lingüísticos y literarios del Noroeste, coords. E. Mendoza et al., Universidad Autónoma de Sinaloa, Culiacán, 2008, t. 1, pp. 11-32. En la película de dibujos animados se utilizan seis versiones: "argentino", "mexicano", "castellano" y "español neutro" para los doblajes; y "español" y "castellano" para los subtítulos. Desde el punto de vista fonético, predomina sin duda la norma alfa, que se usa en los doblajes al mexicano y al español neutro. Llama la atención el hecho de que la versión subtitulada en español corresponda, desde el punto de vista léxico, al español neutro y al mexicano. En mi art. menciono también la actitud separatista de España a partir del doblaje de la película de Disney La bella y la bestia, de principios de los años noventa del siglo pasado.
} 


\section{APÉNDICE}

\section{DATOS DE LOS PROGRAMAS DEPORTIVOS DE RADIO}

Nota: Además de los conductores registrados en la lista, también aparecen en los programas las voces de reporteros, invitados y otros. Sólo se revisó la nacionalidad de los locutores oficiales, pero se consignó la aparición del resto de las voces en los archivos de trabajo, los cuales no fueron considerados en las transcripciones fonéticas. Los países aparecen en orden alfabético en la lista. Todas las grabaciones se realizaron durante los años 2008-2009 y 2015 en la misma estación de radio, excepto donde se indican otros datos.

Argentina: Radio Continental (Buenos Aires 590 AM); www.continental.com. ar/envivo.htm; programa: "Competencia”; conductores: Alejandro Apo y Matías Canillán.

Bolivia: Radio Panamericana (La Paz 96.1 FM); www.panamericana-bolivia.com/; www.megalink.com/radio/panamericana/index.php; programa: "Panamericano Deportivo"; conductores: Ramiro Sánchez y Pablo Loza.

Chile: Radio Universidad de Chile (Santiago 102.5 FM); www.radio.uchile.cl; programa: "Hola Deportes"; conductores: Marco Sotomayor, Juan Antonio Belmar y Pedro Pavlovic.

Colombia: Radio Caracol (Bogotá 810 AM); www.caracol.com.co; programa: "El Pulso del Fútbol"; conductores: Hernán Peláez e Iván Mejía.

Costa Rica: Monumental (San José 93.5 FM); www.monumental.co.cr/parrilla. aspx; programa: "Al Pie del Deporte"; conductor: Everardo Herrera; grabación realizada en 2008. Radio Actual (San José 107.1 FM); www.radioactualfm. com/en-vivo/; programa: "Al Pie del Deporte"; conductor: Everardo Herrera; grabación realizada en 2015 .

Cuba: Radio Rebelde (La Habana 96.70 FM); www.radiorebelde.com.cu/programacion.htm; programa: "Record"; conductor: Magdiel Pérez Labrada.

Ecuador: CRE Satelital Ecuador (Quito 560 AM); www.cre.com.ec; programa: "Los Cronistas"; conductores: Diego Arcos, Kenni Castro, Gustavo Yépez y Pedro Merchán.

El Salvador: Milenio (San Salvador 92.1 FM); radiomilenio.net/index. php?option=com_content\&task=view\&id=12\&Itemid=28; programa: "Noticiario deportivo"; conductor: Mauricio Rivas; grabación realizada en 2008. Radio Nacional de El Salvador (San Salvador 96.9 FM); 66.7.198.72:8000/ radionacional.org/; programa: "Todo deportes"; conductor: Marco Rodríguez; grabación realizada en 2015.

España: Rock \& Gol (Madrid - Radio Popular); www.rockandgol.net/programacion.php; programa: "Rock \& Gol Deportes"; conductor: Luis Munilla; grabación realizada en 2008. Cadena SER (Madrid); play.cadenaser.com/ 
programas/; programa: "SER Deportes"; conductor: Francisco José Delgado; grabación realizada en 2015.

Guatemala: Emisoras Unidas (Guatemala 89.7 FM); radio.emisorasunidas. com/programacion.php; programa: "Súper Deportivo"; conductores: Kike Rodríguez y Epson Aldana.

Honduras: Radio Inter (Tegucigalpa 91.9 FM); www.inter919.com; programa: "Panorama Deportivo"; conductores: Víctor "Tito" Handal y Jesús Vélez Banegas.

México: Reporte (México 98.5 FM); http://www.reporte.com.mx/; programa: "Reporte deportivo"; conductores: Juan Carlos Veraza, Roberto Sosa y Gerardo Valtierra.

Nicaragua: Radio Sport (Managua, 99.3 FM); http:/ / radiosultana.com/radiosultana/index.php?option=com_content\&task=view\&id=17\&Itemid=36; programa: "Hit Deportivo"; conductor: Edgar Estrada; grabación realizada en 2008. La 580 AM (Managua, 580 AM); www.la580.com; programa: "Play off"; conductores: Enrique Armas, Edgard Rodríguez, Edgar Estrada e Iván Centeno; grabación realizada en 2015.

Panamá: W Radio (Panamá 94.5 FM); http://www.wradio.com.pa/programas.asp?idgrp=363628; programa: "Frecuencia deportiva"; conductores: David Salayandía, Ramón "Monchi” Webster y Santiago Gil Sam.

Paraguay: Radio Nanduti (Asunción 1020 AM); www.nanduti.com.py; programa: "Puro Fútbol"; conductores: Rubén Darío Da Rosa, Gabriel Cazenave y Robert Singer; grabación realizada en 2008. Radio Nanduti (Asunción 1020 AM); www.nanduti.com.py; programa: "Magazine Deportivo"; conductores: Marcelo Orue y Roberto "Tito” González; grabación realizada en 2015.

Perú: Radio Ovación (Lima 620 AM); www.ovacion.com.pe/Radio/index. htm; programa: "La oral deportiva"; conductor: José Valdeiglesias.

Puerto Rico: Noti Uno (San Juan, 630 AM); http:/ /www.notiuno.com/programacion/; programa: "A batazo limpio"; conductor: Carlos A. Valero Viruet.

República Dominicana: Monumental (Santo Domingo 100.3 FM); http:/ /www. monumentalfm.com/programacion.html; programa: "Las deportivas"; conductor: Osvaldo Matías.

Uruguay: Radio Universal (Montevideo 970 AM); www.22universal.com; programa: "El Oral deportivo"; conductores: Alberto Kesman, Dr. Ariel Delbono y Enrique Yannuzzi.

Venezuela: Unión Radio Deportes (Caracas 1090 AM); unionradio.planetatv. com; programa: "Deportivas Unión Radio"; conductores: Fernando Arreaza, John Carrillo e Iván Arteaga. 
